\title{
Phenotypic characterisation of Saccharomyces spp. yeast for tolerance to stresses encountered during fermentation of lignocellulosic residues to produce bioethanol
}

\author{
Tithira T Wimalasena ${ }^{1 \dagger}$, Darren Greetham ${ }^{1 \dagger}$, Marcus E Marvin², Gianni Liti ${ }^{3}$, Yogeshwar Chandelia', Andrew Hart', \\ Edward J Louis ${ }^{2}$, Trevor G Phister ${ }^{1,5}$, Gregory A Tucker ${ }^{1}$ and Katherine A Smart ${ }^{1,4^{*}}$
}

\begin{abstract}
Background: During industrial fermentation of lignocellulose residues to produce bioethanol, microorganisms are exposed to a number of factors that influence productivity. These include inhibitory compounds produced by the pre-treatment processes required to release constituent carbohydrates from biomass feed-stocks and during fermentation, exposure of the organisms to stressful conditions. In addition, for lignocellulosic bioethanol production, conversion of both pentose and hexose sugars is a pre-requisite for fermentative organisms for efficient and complete conversion. All these factors are important to maximise industrial efficiency, productivity and profit margins in order to make second-generation bioethanol an economically viable alternative to fossil fuels for future transport needs.

Results: The aim of the current study was to assess Saccharomyces yeasts for their capacity to tolerate osmotic, temperature and ethanol stresses and inhibitors that might typically be released during steam explosion of wheat straw. Phenotypic microarray analysis was used to measure tolerance as a function of growth and metabolic activity. Saccharomyces strains analysed in this study displayed natural variation to each stress condition common in bioethanol fermentations. In addition, many strains displayed tolerance to more than one stress, such as inhibitor tolerance combined with fermentation stresses.

Conclusions: Our results suggest that this study could identify a potential candidate strain or strains for efficient second generation bioethanol production. Knowledge of the Saccharomyces spp. strains grown in these conditions will aid the development of breeding programmes in order to generate more efficient strains for industrial fermentations.
\end{abstract}

Keywords: Saccharomyces spp., Phenotypic microarray, Bioethanol, Fermentation

\section{Background}

It is generally recognised that 'renewable' forms of energy, such as those generated from lignocellulosic biomass, will become increasingly important. Currently the production of liquid biofuels by fermentation has focussed on the conversion of hexose sugars to form bioethanol [1], where

\footnotetext{
* Correspondence: katherine.smart@sabmiller.com

${ }^{\dagger}$ Equal contributors

'Bioenergy \& Brewing Science, School of Biosciences, Sutton Bonington Campus, University of Nottingham, Loughborough, Leicestershire LE12 6RD, UK

${ }^{4}$ Current address: SABMiller plc, SABMiller House. Church Street West, Woking, Surrey GU21 6HS, UK

Full list of author information is available at the end of the article
}

hexose carbohydrates are released from sucrose in crops such as sugar cane [2,3], or from starch. However, use of these biomass resources has met criticism and an increased interest in non-starch or cane biomass conversion $[4,5]$.

Bioethanol produced from lignocellulosic residues may be more socioeconomically prudent, however, the technical block to this approach remains the efficient conversion of hexose and pentose sugars into ethanol or alternative liquid biofuels [6]. Saccharomyces cerevisiae is widely employed for the commercial production of bioethanol from hexose sugars. However, S. cerevisiae strains are unable to efficiently utilise pentose sugars [7], despite containing a xylose utilisation pathway [8] and an arabinose 
metabolic pathway [9]. This restricts the potential use of non-recombinant strains of $S$. cerevisiae for the production of bioethanol from lignocellulosic feedstocks [10-13].

Saccharomyces spp. are attractive because of their capacity to produce ethanol. Their relatively high tolerance to osmotic stress and ethanol and their tolerance to anaerobic conditions are characteristics that are suitable for large-scale fermentation [14]. Much less is known about their capacity to tolerate the inhibitors released during the formation of lignocellulosic hydrolysates $[15,16]$. The Saccharomyces spp. (formerly termed Saccharomyces sensu stricto) complex consists of seven closely related but distinct species; S. cerevisiae, S. paradoxus, S. mikatae, S. kudriavzevi S. arboricolus, and S. uvarum, [17-20]. Within $S$. cerevisiae there is an enormous amount of genetic variability that is believed to result from its geographical movement by man along with outcrossing to generate strains with mosaic genomes [21,22]. Five clean lineage strains (West African, Wine European, Sake, North American and Malaysian) of S. cerevisiae that are representative of specific genomic clades have been identified [22] and engineered to enable genetic tractability [23-25].

Research has shown that approaches such as phenotypic selection of natural isolates, breeding programmes assisted by technological tests, quantitative trait locus (QTL) introgression and genetic engineering have been successful in strain development for the food and beverage industries $[1,26,27]$. In addition, interbreeding is very common among Saccharomyces strains giving rise to naturally occurring novel hybrid strains that have been identified in the brewing and wine industries [28,29]. All species within the Saccharomyces spp. complex can be mated to form hybrid diploids and these can subsequently be utilised to produce bioethanol. Therefore, phenotypic screening of Saccharomyces strains can be an important initial tool for isolating a strain with desirable traits for efficient bioethanol fermentation.

Here, we have screened Saccharomyces spp. strains and selected hybrids for phenotypic variation in terms of tolerance to osmotic stress, temperature $\left(30^{\circ} \mathrm{C}-40^{\circ} \mathrm{C}\right)$, increased ethanol concentration and inhibitory compounds released through the pre-treatment of lignocellulosic biomass and utilisation of hexose and pentose sugars. By coupling phenotypic and genetic analysis, selective breeding and evolutionary engineering, novel yeast strains can be produced with inherent properties for improving industrial processes such as bioethanol production from lignocellulosic wastes [30-32].

The aim of this study was to identify strains capable of tolerating the stress and inhibitor conditions associated with lignocellulosic bioethanol fermentation. A selection of Saccharomyces strains were chosen that had been isolated from natural habitats, wine, beer, baking or clinical backgrounds [22].

\section{Results}

Phenotypic variation and ranking of responses of yeast strains to stress

Ninety strains of Saccharomyces spp. (89 formerly termed Saccharomyces sensu stricto yeast and the phylogenetically distinct $S$. castelli sensu stricto group outsider) were screened. Phenotypic responses to parameters encountered during the fermentation of lignocellulosic hydrolysates were tested. These included the utilisation of hexose and pentose sugars, resistance to conditions within bioreactors, such as osmotic, ethanol and temperature stress and resistance to phenolic and aromatic inhibitory compounds formed during the steam explosion of lignocellulosic waste. Phenotypic variation of the strains to these stresses was observed and ranked according to the impact on metabolic output, defined here as percentage of redox signal intensity of control (Additional file 1: Figure S1).

\section{Utilisation of hexose and pentose sugars by Saccharomyces spp. strains}

Hydrolysates derived from LCM contain hexose and pentose sugars, [33], metabolic output (defined here as the detection of conversion of a redox sensitive dye from an oxidised to reduced state) on glucose, xylose and arabinose was measured. These sugars are the most abundant in LCM hydrolysates after pre-treatment of lignocellulose [34]. There was measurable metabolic output when utilising glucose for all strains in this study with variation observed between the strains (35-75 redox signal intensity) after 48 hours incubation (Additional file 2). Assays for utilisation of the pentose sugars, xylose and arabinose, revealed that the majority of the strains exhibited very poor metabolic output $(<20$ redox signal intensity) after $50 \mathrm{hrs}$ incubation (Additional file 1: Figure S1). The reference yeast used for these experiments, S288C, has previously been reported as being a poor pentose utilising yeast [12] and we failed to observe significant improvements in metabolic output when utilising either xylose or arabinose (Additional file 1: Figure S1). There are a few strains which to a degree exhibited metabolic output when utilising xylose such as S. cerevisiae YS2, or when utilising arabinose such as S. cerevisiae NCYC110 (Figure $1 \mathrm{~A}$ and $1 \mathrm{C}$ ). However, assays comparing these strains with known pentose utilisation yeast (Candida shehatae or Scheffersomyces spitis) highlighted the poor pentose utilisation by Saccharomyces spp. (data not shown). Saccharomyces spp. vary in their biochemical profiles [35] and variation in metabolic output in general may have accounted for some of the differences in xylose utilisation between strains. However, there was no correlation observed between glucose utilisation and xylose utilisation $(\mathrm{R}=0.081)$. Saccharomyces spp. strains shared similarities in the utilisation of xylose and arabinose $(R=0.41)$. However, the majority of the strains 


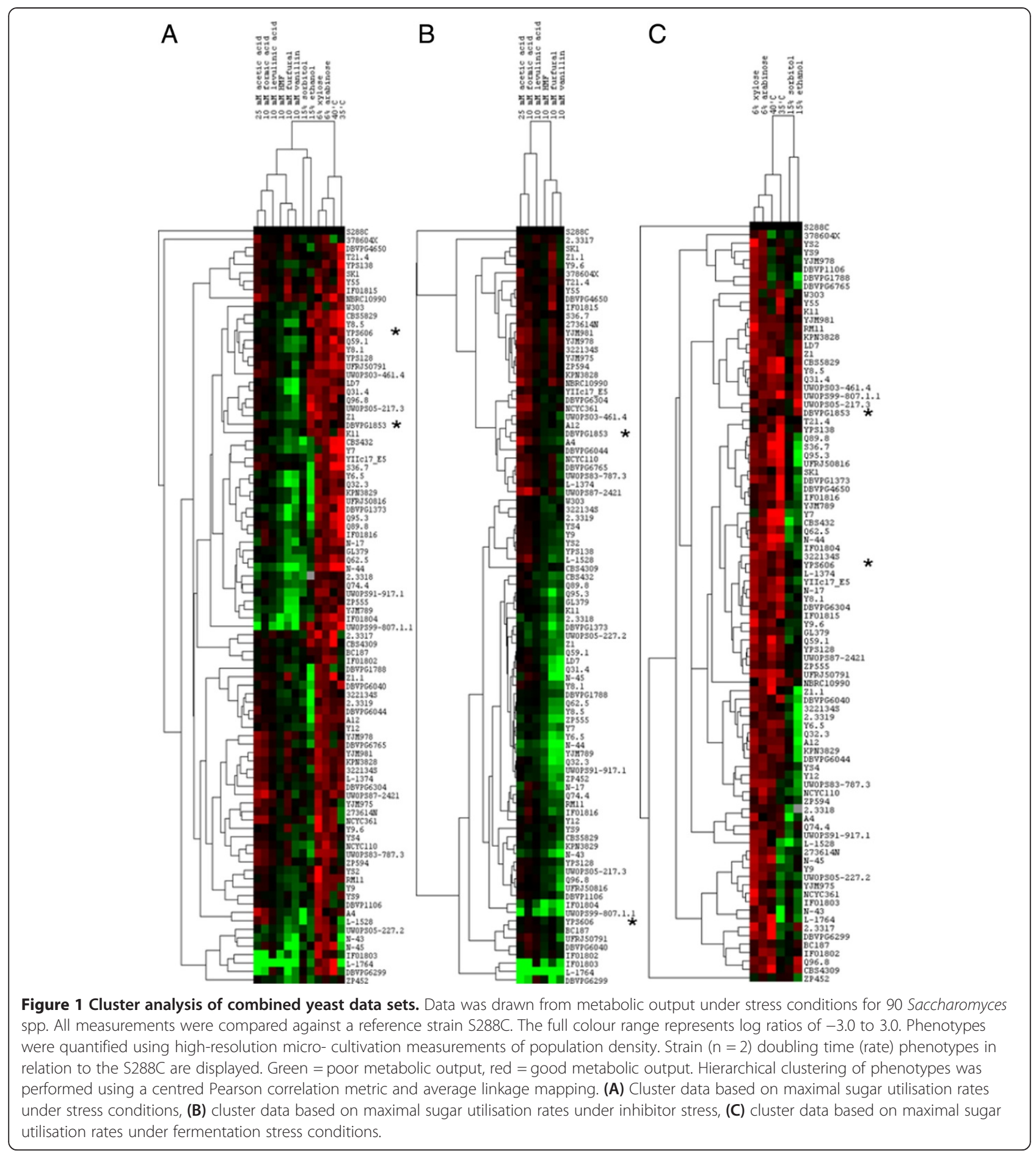

analysed showed a lack of significant xylose or arabinose utilisation.

\section{Phenotypic variation to stress inherent to fermentations within Saccharomyces spp. strains}

Constant exposure to high temperatures or severe osmotic stress has been used to identify tolerant yeast strains [36].
Tolerance to osmotic, ethanol or temperature stress was characterised by profound phenotypic variation between yeast strains (Figure 1A-C). Using the ranking system, S. uvarum (DBVPG6299), S. paradoxus (Q74.4) and S. arboricolus (2.3317) strains were identified as being osmotically tolerant when compared with other strains used in the study (Figure 2A and Table 1). In general, $S$. kudriavzevii spp. displayed higher sensitivity to osmotic 

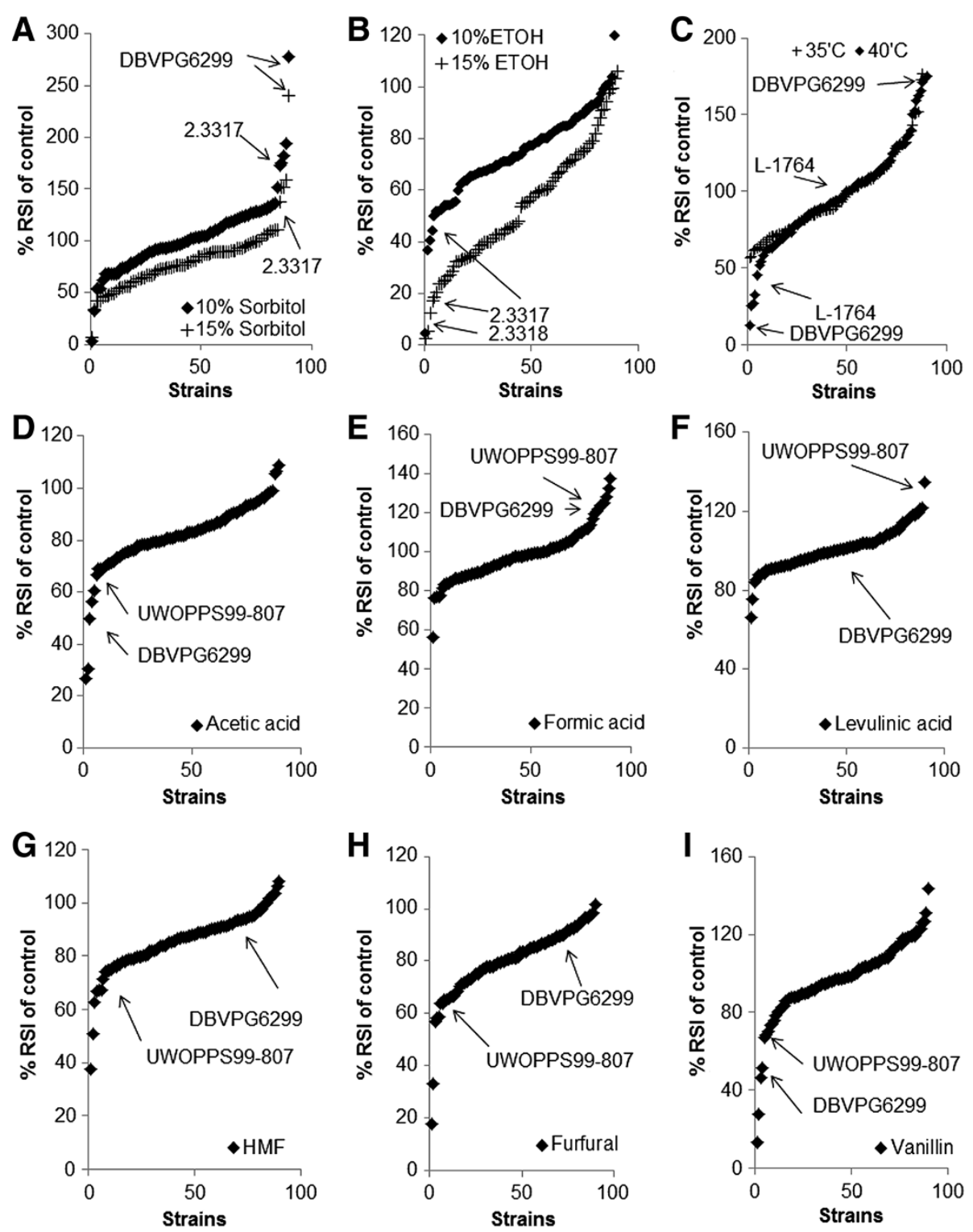

Figure 2 Phenotypic microarray analysis (redox signal intensity) of Saccharomyces spp. for the effect of fermentation stress (temperature, osmotic and ethanol) and inhibitors at 50 hrs. Yeast were grown in minimal medium containing $6 \%$ glucose at $30^{\circ} \mathrm{C}$ and metabolic activity assessed at 50 hrs time point. Results are plotted as\% of RSI (redox signal intensity) where metabolic output in the presence of defined stresses were compared to unstressed conditions. (A) $10 \%$ and $15 \%$ sorbitol, (B) $10 \%$ and $15 \%$ ethanol, (C) temperature $\left(35^{\circ} \mathrm{C}\right.$ and $\left.40^{\circ} \mathrm{C}\right)$ (D) $25 \mathrm{mM}$ acetic acid, (E) $10 \mathrm{mM}$ formic acid, (F) $10 \mathrm{mM}$ levulinic acid, (G) $10 \mathrm{mM}$ furfural, (H) $10 \mathrm{mM}$ HMF and (I) 10 mM vanillin, on sugar utilisation on Saccharomyces spp. ranked from sensitive to tolerant strains. Selected yeast strains have been highlighted as appropriate.

stress when compared with other Saccharomyces spp. yeast strains.

Screening for tolerance to $15 \%$ ethanol, six of the ten most tolerant strains (determined by observable metabolic output under stress conditions when compared with other yeast strains in this study) were $S$. cerevisiae strains isolated from either a clinical or a fermentation background. S. arboricolus strains (2.3317 and 2.3318) displayed the highest sensitivity to ethanol when compared with other yeast in this study (Figure 2B), however, 2.3317 is tolerant to osmotic (sorbitol) stress when compared with the other S. arboricolus strains (Figure 1A).

For temperature stress there was a reduction in metabolic output at $40^{\circ} \mathrm{C}$ (using data from assays at $30^{\circ} \mathrm{C}$ as a control) for the majority of strains when compared with output at $30^{\circ} \mathrm{C}$ and $35^{\circ} \mathrm{C}$. Interestingly, most of the $S$. cerevisiae isolated from either a clinical or baking backgrounds displayed tolerance to $40^{\circ} \mathrm{C}$. However, $S$. kudriazevii strains (with the exception of strain IFO1803) were sensitive to increasing temperature; they displayed a 
Table 1 Ranking of yeast strains for tolerance to stress conditions in bioethanol fermentation

\begin{tabular}{|c|c|c|c|c|c|c|c|c|c|c|c|c|c|c|c|c|c|c|c|c|}
\hline \multicolumn{3}{|l|}{ Sorbitol } & \multicolumn{2}{|c|}{ Ethanol } & \multicolumn{2}{|l|}{$35^{\prime} \mathrm{C}$} & \multicolumn{2}{|l|}{$40^{\prime} \mathrm{C}$} & \multicolumn{2}{|c|}{ Acetic acid } & \multicolumn{2}{|c|}{ Formic acid } & \multicolumn{2}{|c|}{ Furfural } & \multicolumn{2}{|l|}{ HMF } & \multicolumn{2}{|c|}{ Vanillin } & \multicolumn{2}{|c|}{ All stress } \\
\hline Sensitive & S. $p$ & $\mathrm{~N}-17$ & S. $a$ & 2.3318 & S.C & DBVPG1853 & S. $p$ & CBS432 & S. $a$ & 2.3317 & S.C & Y12 & S.C & $\mathrm{Y} 12$ & S. cas & CBS4309 & S.C & $\mathrm{Y} 12$ & S.C & DBVPG1853 \\
\hline & S. C & W303 & S. $a$ & 2.3317 & S. C & $\begin{array}{l}\text { UWOPS83- } \\
787.3\end{array}$ & S. $k$ & NBRC10990 & S.C & CBS4309 & S. $p$ & $\mathrm{~N}-17$ & S. cas & CBS4309 & S.C & Y12 & S. cas & CBS4309 & S.u & L-1764 \\
\hline & S. $k$ & ZP452 & S. $p$ & LD7 & S. C & $\begin{array}{l}\text { UWOPSO3- } \\
461.4\end{array}$ & S. $b$ & DBVPG6045 & S.C & $\mathrm{Y} 12$ & S. C & YJM981 & S. $p$ & $\mathrm{~N}-45$ & S. C & 3221345 & S. $a$ & 2.3317 & S. $p$ & Q32.3 \\
\hline & S.C & DBVPG1788 & S.p & UFRJ50816 & S.C & Y9 & S. $a$ & 2.3317 & S. $p$ & Q74.4 & S.C & Yllc17_E5 & S. $p$ & Q74.4 & S. $m$ & IFO1815 & S. $p$ & Q74.4 & S. $p$ & UFRJ50816 \\
\hline & S. $p$ & Q32.3 & S. $p$ & Q32.3 & S. $p$ & Y7 & S. $b$ & $L-1764$ & S. $b$ & $\begin{array}{l}\text { UWOPS99- } \\
807.1 .1\end{array}$ & S. cas & CBS4309 & S. C & Yllc17_E5 & S.C & Yllc17_E5 & S. C & Yllc17_E5 & S.C & $\begin{array}{l}\text { UWOPS83- } \\
787.3\end{array}$ \\
\hline \multirow[t]{12}{*}{ Tolerant } & S.C & YJM789 & S.C & YPS606 & S.C & DBVPG6040 & S. C & YS2 & s. $b$ & DBVPG6045 & S.C & $\begin{array}{l}\text { UWOPS83- } \\
787.3\end{array}$ & S.C & RM11 & S. C & DBVPG6040 & S. C & Y9 & S. $p$ & $\begin{array}{l}\text { UWOPS91- } \\
917.1\end{array}$ \\
\hline & S. C & SK1 & S. $p$ & Q96.8 & S. $p$ & Q96.8 & S. $p$ & Q89.8 & S. $p$ & YPS138 & S. $b$ & L-1764 & S. $a$ & 2.3317 & S. $p$ & Y6.5 & S. $p$ & GL379 & S. $p$ & Q89.8 \\
\hline & S. $b$ & $\begin{array}{l}\text { UWOPS99- } \\
807.1 .1\end{array}$ & S.C & YJM975 & S.C & $378604 x$ & S. $p$ & $\begin{array}{l}\text { UWOPS91- } \\
917.1\end{array}$ & S. $m$ & IFO1816 & S. C & S288C & S. C & YJM978 & S. C & YJM978 & S. $C$ & YPS606 & S. C & YPS606 \\
\hline & S.C & $378604 X$ & S. $b$ & ZP555 & S. $p$ & IFO1804 & S. C & YJM975 & S.p & UFRJ50816 & S.C & YJM978 & S. $b$ & L-1764 & S.C & YS9 & S.C & S288C & S.C & $378604 x$ \\
\hline & S. $p$ & DBVPG6304 & S. C & Y12 & S. $a$ & 2.3317 & S. C & DBVPG6044 & S. $p$ & Y8.1 & S. $k$ & IFO1803 & S. C & YPS128 & S. C & L-1374 & S. C & $\begin{array}{l}\text { UWOPS87- } \\
2421\end{array}$ & S. C & YJM975 \\
\hline & S.C & S. cerevisiae & & & & & & & & & & & & & & & & & & \\
\hline & S. $p$ & S. paradoxus & & & & & & & & & & & & & & & & & & \\
\hline & S. $k$ & S. kudriavzevii & & & & & & & & & & & & & & & & & & \\
\hline & S. u & S. uvarum & & & & & & & & & & & & & & & & & & \\
\hline & S. $a$ & S. arboricolus & & & & & & & & & & & & & & & & & & \\
\hline & S. cas & S. castelli & & & & & & & & & & & & & & & & & & \\
\hline & S. $m$ & S. mikatae & & & & & & & & & & & & & & & & & & \\
\hline
\end{tabular}

Ranking of Saccharomyces spp. strains for stress conditions analysed in this study. The five most tolerant and sensitive strains for each stress conditions are shown in this table. The full list of strains is available in Additional file 1: Figure S1. 
reduction in metabolic output at 35 and $40^{\circ} \mathrm{C}$ when compared with $30^{\circ} \mathrm{C}$.

The metabolic output at $35^{\circ} \mathrm{C}$ was increased for $S$. uvarum strains (DBVPG6299 and UWOPS99-807.1.1) used in this study. The metabolic output of the $S$. uvarum strain (L1764) was decreased at $35^{\circ} \mathrm{C}$ compared to $30^{\circ} \mathrm{C}$. Growth analysis of these strains at $35^{\circ} \mathrm{C}$ and $40^{\circ} \mathrm{C}$ confirmed the results obtained from PM analysis (Additional file 1: Figure S1).

\section{Phenotypic variation to inhibitors within Saccharomyces spp. strains}

The presence of inhibitors in lignocellulosic fermentations has been shown to have a profound effect on the sugar utilisation and viability of $S$. cerevisiae (Greetham, et al., unpublished data). Saccharomyces spp. were assayed for the effect of acetic acid, formic acid, levulinic acid, HMF, furfural and vanillin on metabolic output using inhibitory concentrations. The concentrations tested for acetic acid, furfural and formic acid have been shown to be released during pre-treatment of lignocellosic material [34] and for HMF, vanillin and levulinic acid have been previously shown to inhibit yeast $[37,38]$ (Greetham et al., unpublished).

In general, S. uvarum strains, such as (DBVPG6299), screened in this study were sensitive to inhibitory compounds. However, there were exceptions such as UWOPS99-807.1.1, which exhibited tolerance to both formic acid and levulinic acid (Figure 2E and 2F). Furfural tolerant strains were either $S$. cerevisiae or the closely related $S$. paradoxus (Figure 2D-2I). Therefore, the general trends in tolerance/sensitivity followed species designations.

\section{Hierarchical clustering followed species and trait boundaries} Utilising a phenotypic microarray assay, differences between yeast species in terms of tolerance to stresses inherent to fermentations have been defined. Defining tolerance to more than one stress has allowed for the identification of robust yeast for future bioethanol fermentations (Table 1). However, the data was analysed further in an attempt to discern whether phenotypic response to stress is general, or whether or not specific yeast clades cluster together. Hierarchical clustering of Saccharomyces spp. based on phenotypic trait profiles (Figure 1A and 1B) was compared with the haploid reference laboratory strain S. cerevisiae S288C. The reference strain displayed sensitivity to stresses inherent to fermentations and hence the majority of strains in the cluster analysis appeared red (more tolerant) (Figure 1A and 1B). The reference yeast, $\mathrm{S} 288 \mathrm{C}$ is one of the more sensitive strains to the presence of inhibitory compounds when compared with other yeast analysed in this study. However, it was one of the more thermo-tolerant strains (displaying metabolic activity at 35 or $40^{\circ} \mathrm{C}$ when compared with metabolic output at $30^{\circ} \mathrm{C}$ ) and was phenotypically distinct from the other yeast in response to stress (Figure 1A-C). S288C is known to be phenotypically distinct from other $S$. cerevisiae strains and this has been previously reported [39].

The phenotypic microarray results for pentose sugars clustered with the results for temperature, ethanol or osmotic stress (Figure 1A and 1C). On the other hand, response to inhibitory compounds clustered separately from the phenotypic response to fermentation stress (osmotic, temperature and ethanol). Inhibitory compounds (such as weak acids or furanic compounds) clustered together (Figure 1A and 1B).

There was a clearly observed dichotomy at the UWO PS99-807.1.1/2.3317 boundary (Figure 1A). Strains S288C to UWOPS99-807 are characterised by similar phenotypic responses to inhibitory compounds (with the exception of formic acid), are sensitive to ethanol and displayed better thermo-tolerance than the reference strain. Strains 2.3317 to DBVPG1106 were characterised as being more tolerant to inhibitory compounds, osmotic stress and temperature stress than S288C, but more sensitive to the presence of ethanol. Strains on Figure 1A from A4-ZP452 are inhibitor (including formic acid) and/or temperature sensitive.

We also observed a boundary between weak acid tolerance/furanic/phenolic tolerance and weak acid/furanic/ phenolic sensitivity (Figure 1B). Strains from 2.3317 to W303 were more tolerant to the presence of weak acids than those from W303 to DBVPG6299. However, strains W303 to DBVPG6299 were more sensitive to the presence of furanic or phenolic compounds than the reference strain, S288C.

However, S. uvarum and S. kudriavzevii clustered together when the effect of stress on was determined, they share similar responses to inhibitor and temperature stress (Figure $1 \mathrm{~A}$ and $1 \mathrm{~B}$ ).

\section{Confirmation of phenotypic microarray strain assessments using mini-fermentation analysis}

The objective of this study was to identify potential candidate strains for efficient second generation bioethanol fermentations. Phenotypic variation to stress as mentioned earlier was ranked according to tolerance to the various stresses, allowing the identification of tolerant yeast for any tested stress condition (Table 1). From the 90 strains screened and ranked S. cerevisiae strain, YPS 606, was selected as a strain with general tolerance to stress with the $S$. cerevisiae strain, DBVPG1853, is the most sensitive strain after 48 hours of the assay (Additional file 1: Figure S2). The latter is also phenotypically similar to $\mathrm{S} 288 \mathrm{C}$, which is also sensitive to stress (Figure 1A-C).

These two strains were used in fermentation experiments, with fermentation progression monitored by measuring weight loss over time. This has been shown to 
correlate with sugar utilisation [40] in the presence of inhibitory compounds. These were compared to fermentations of unstressed controls at $35^{\circ} \mathrm{C}$. Fermentations with YPS606 were not affected by the presence of inhibitors when compared with controls (Figure 3A). However, presence of inhibitory compounds did slow a fermentation using DBVPG1853 by approximately 2 hrs when compared with unstressed controls (Figure 3B).

\section{Trait hereditary in diploid hybrids confers tolerance compared with parental sensitivity}

Saccharomyces strains (YPS128, Y12, Y55 and IFO1803) were used to generate diploid hybrids. By assessing tolerance to stress, it was observed that YPS128 and Y12 exhibited different sensitivities to the presence of inhibitory compounds (defined as the number of more tolerant yeast strains assayed for in this study). Y55 (S. cerevisiae) and IFO1803 (S. kudriavzevii) exhibit different tolerance to the presence of inhibitory compounds, temperature stress, or ethanol stress (Additional file 1: Figure S3 and Additional file 2). These hybrid strains and their homozygous parents were then assessed for tolerance to a range of fermentation stress conditions using PM assays.

The tolerances of a $S$. cerevisiae cross between YPS 128 and Y12 to formic acid, osmotic stress and high temperature was measured. Under unstressed control conditions, metabolic output of the heterozygous hybrid diploid (YPS $128 \times$ Y12) was an intermediate of the metabolic output of either haploid parent (Figure 4A). There was a variation in the phenotypic response to stress observed when compared with either parent. When under osmotic or temperature stress, the hybrid diploid had the same profile as the sensitive parent Y12 (Figure 4B and 4D). Y12 was highlighted as being sensitive to the presence of inhibitory compounds in the initial screen, whereas YPS128 had a more tolerant phenotype (Additional file 1: Figure S1). The hybrid diploid was more tolerant to stress induced by inhibitory compounds than Y12 (Figure 4C).

We wanted to observe how a heterozygous hybrid diploid resulting from a cross between a non-S. cerevisiae strain that was sensitive to stress (S. kudriavzevii 1803) and a stress tolerant $S$. cerevisiae strain (Y55) would behave under our test conditions. It was observed that under unstressed control conditions, the hybrid diploid displayed an identical metabolic output to the parental strain Y55 (Figure 4E). The hybrid diploid was more sensitive to osmotic or acetic acid induced stress than Y55, but was more tolerant than the S. kudriavzevii strain 1803 (Figure 4F and 4G). There was little variation observed under temperature stress between the parents and hybrid (Figure 4H).

\section{Discussion}

Industrial scale fermentations always favour yeast with efficient fermentation capabilities, particularly S. cerevisiae and the closely related S. uvarum [41]. Yeast cells encounter osmotic stress due to high sugar and solute concentrations at the beginning of fermentation [42]. During fermentation additional stresses such as the accumulation of ethanol also become relevant [43]. In addition, during second generation bioethanol fermentations organisms are exposed to inhibitory compounds released by the pre-treatment of lignocellulosic material. Complex and advanced strategic approaches, based on genetic engineering strategies have been utilised to increase the innate tolerance of yeast cells to inhibitory compounds [7]. Understanding the mechanisms of resistance to these compounds are commercially attractive [44]. The possibility of using natural selection and breeding programmes to develop non-GM strains of $S$. cerevisiae that can efficiently
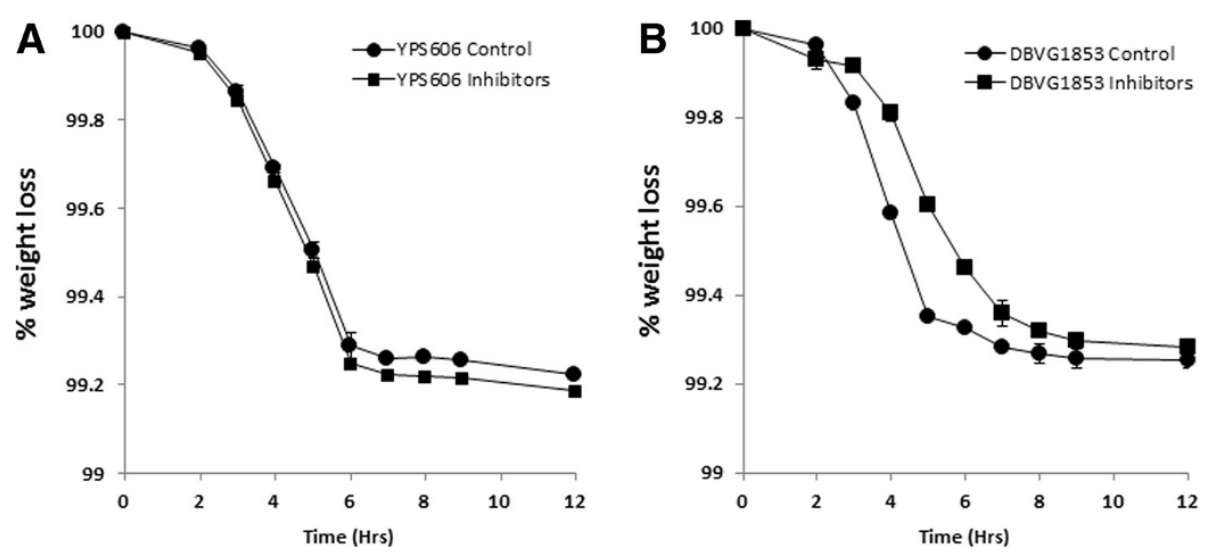

Figure 3 Performance of yeast strains during fermentations in the presence of inhibitory compounds. Comparison of sensitive (DBVPG1853) and tolerant (YPS606) S. cerevisiae strains to stress, fermentation kinetics analysis using mini-fermenters on $4 \%(\mathrm{~W} / \mathrm{V})$ glucose and inhibitors (10 mM acetic acid, $5 \mathrm{mM}$ formic acid, $5 \mathrm{mM}$ levulinic acid, $5 \mathrm{mM} \mathrm{HMF}, 5 \mathrm{mM}$ furfural and $5 \mathrm{mM}$ vanillin in combination) at $35^{\circ} \mathrm{C}$ using (A) S. cerevisiae YPS606 and (B) DBVPG1853. Each data point represents mean value of biological triplicate experiments with SD error bars. 

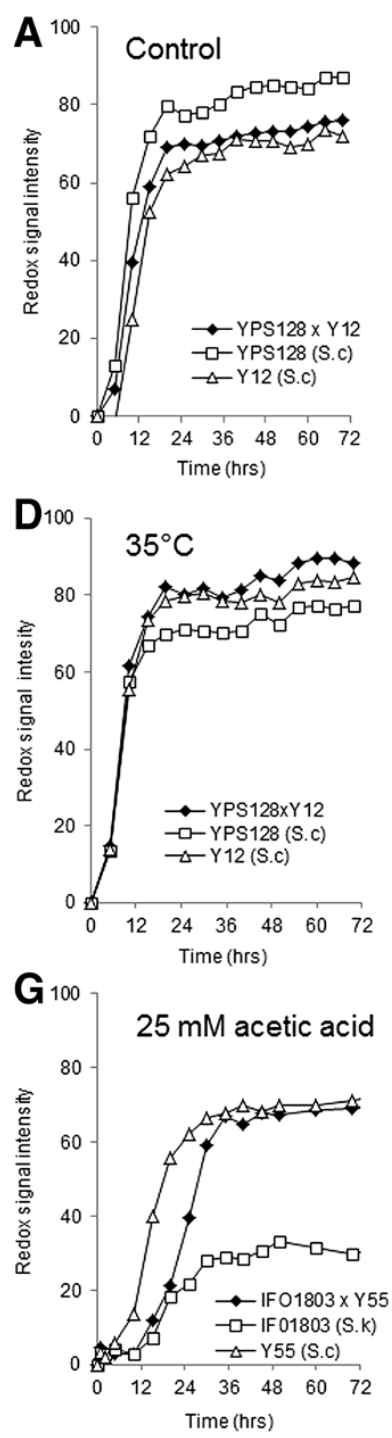
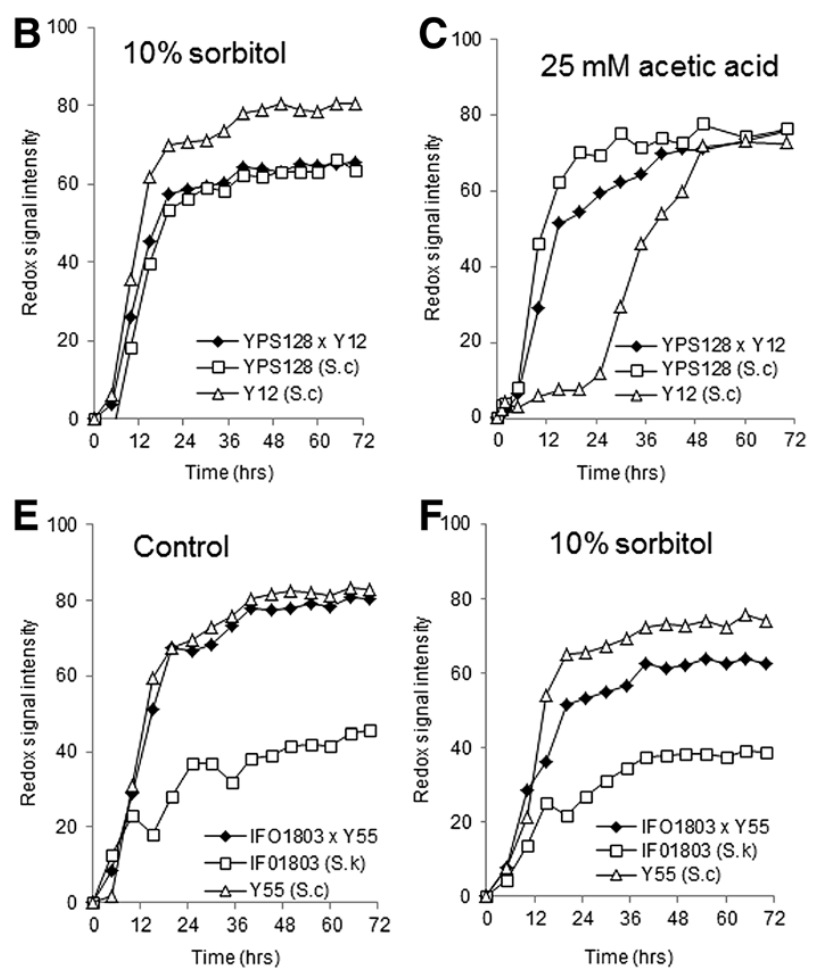

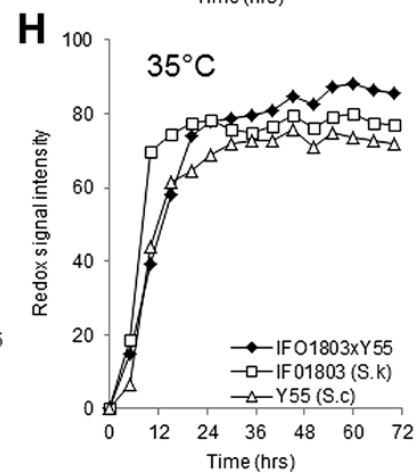

Figure 4 Phenotypic microarray analysis (redox signal intensity) of hybrids. The response of hybrid diploids to stress was compared with the parental stains S. cerevisiae (Y21) and S. cerevisiae (YPS128) (A-E) and S. cerevisiae (Y55) and S. kudriavzevii (IFO1803) (F-I) Metabolic output for S. cerevisiae (Y12), S. cerevisiae (YPS128) and a diploid cross (Y12 $\times$ Y128) for (A) control $\left(6 \%(\mathrm{~W} / \mathrm{V})\right.$ glucose at $\left.30^{\circ} \mathrm{C}\right),(\mathbf{B}) 6 \%$ glucose and $10 \%(\mathrm{~W} / \mathrm{V})$ sorbitol, (C) $6 \%$ glucose and $25 \mathrm{mM}$ acetic acid (D) $6 \%$ glucose at $35^{\circ} \mathrm{C}$. Metabolic output for S. cerevisiae (Y55) and S. kudriavzevii (IFO1803) and a diploid cross (Y55 $\times$ IFO1803) for (E) control (6\% (W/v) glucose at 30 $\mathrm{C}$ ), (F) $6 \%$ glucose and $10 \%(\mathrm{~W} / \mathrm{v})$ sorbitol, (G) $6 \%$ glucose and $25 \mathrm{mM}$ acetic acid and $(\mathrm{H}) 6 \%$ glucose at $35^{\circ} \mathrm{C}$. The data shown is an average of triplicate values with standard deviations shown.

convert hexose and pentose sugars into ethanol under stressful fermentation conditions is being pursued [45] and is ecologically and ideologically attractive.

Screening for xylose utilisation within the Saccharomyces spp. identified strains that have been previously reported as being better utilisers of xylose [12]. In addition S. kudriavzevii, strains have been previously reported as being temperature sensitive $[26,46]$. However, $S$. cerevisiae and S. paradoxus strains were also identified that were able to tolerate high osmotic stress (15\% sorbitol), high ethanol concentrations (15\% ethanol) and higher temperatures $\left(35^{\circ} \mathrm{C}\right.$ and $\left.40^{\circ} \mathrm{C}\right)$. Assays at $35^{\circ} \mathrm{C}$ were characterised by an increase in metabolic output for the majority of S. cerevisiae and S. paradoxus strains. An increase in metabolic output at $35^{\circ} \mathrm{C}$ for $S$. cerevisiae has been reported previously $[26,46]$. It was observed that Saccharomyces strains isolated from clinical backgrounds exhibited the highest tolerance to thermal stress as may be expected. S. uvarum has been identified as thermo-sensitive [47]. However, metabolic output was increased for $S$. uvarum strains (DBVPG6299 and UWOPS99-807.1.1) at $35^{\circ} \mathrm{C}$. Metabolic output for DBVPG6299 was significantly reduced at $40^{\circ} \mathrm{C}$ and this yeast has been previously reported as being temperature sensitive [48]. The metabolic output of the $S$. 
uvarum strain (L1764) decreased at $35^{\circ} \mathrm{C}$ when compared with metabolic output at $30^{\circ} \mathrm{C}$. The phylogenetically distinct $S$. castelli [49] was observed to be thermo-tolerant characterised by increased metabolic output at $40^{\circ} \mathrm{C}$ when compared with other yeast.

Tolerances/sensitivities tended to follow strain designations, for example all $S$. cerevisiae and $S$. paradoxus strains investigated in our study were able to tolerate $10 \%$ ethanol. However, S. cerevisiae isolated from beverage fermentations were the most tolerant to $15 \%$ ethanol. $S$. cerevisiae strains from fermentation backgrounds encounter higher alcohol concentrations, particularly sake fermentations which can generate at least $20 \%(\mathrm{v} / \mathrm{v})$ ethanol [50]. In contrast, S. arboricolus and S. kudriavzevii strains were identified as sensitive to ethanol, supporting reports that these strains were isolated from natural environments and never exposed to an industrial fermentative environment $[20,26]$. In general, yeast isolated from clinical or fermentation backgrounds displayed the highest tolerance to stresses inherent to fermentations. S. arboricolus [20] displayed tolerance to the presence of weak acids when compared with other yeast assayed in this study, however, this yeast sensitive to the toxic effects of ethanol, very little has been published on this yeast and findings here suggest it has some properties which may be relevant for improving bioethanol fermentations.

Clustering revealed that phenotypic response to weak acids, furans, pentose use, osmotic, ethanol and temperature stress all clustered separately and there was an overall strain variation within the Saccharomyces spp. to stress (Figure 1A-1C).

S. cerevisiae strains have been identified as the yeast of choice for efficient bioethanol fermentation due to their ability to convert hexose sugars to high concentrations of ethanol despite the presence of inhibitory compounds in the medium [51]. Using the phenotypic microarray screen, yeast with differing tolerances to stresses were selected and assessed for performance during fermentation. Fermentation profiles correlated with profiles from the phenotypic microarray screen. Thus, tolerant yeast (YPS606) was not inhibited by the presence of an inhibitor stress when compared with control conditions. Interestingly, fermentations using more sensitive yeast (DBVP G1853) under inhibitor stress were characterised by a two hour "slow down" when compared with control.

Hybrid yeast strains are the next step in development of industrial bioethanol yeast, the phenotypes of diploid hybrids generated using selected Saccharomyces spp. strains (S.c.YPS128 $\times$ S.cY12; S.c.Y55 $\times$ S.k.IFO1803) was compared to the metabolic performance of parents under stress conditions. Hybrid strains displayed tolerances similar to one or the other parents or in some instances outperformed either parent. Hybrids displaying tolerance above that of either parent has been noted previously in hybrid diploids [52]. Assessing a cross between Saccharomyces spp. (S. kudriavzevii 1803 x S. cerevisiae Y55), we observed that this diploid was as metabolically active as one of the parents. This hybrid diploid was phenotypically similar to the more tolerant parent under stress conditions, highlighting the potential for cross-breeding between different Saccharomyces spp. for desirable bioethanol strains. Our approach has shown that selected hybrid formation can be a useful tool for the development of novel strains tolerant to the stresses present in bioethanol fermentations.

\section{Conclusions}

In this study, the responses of Saccharomyces spp. strains to the stresses present during bio-ethanol fermentations have been characterised. Considerable natural variation was observed in the stress responses of these yeast and many strains are tolerant to multiple inhibitors. These findings were confirmed when fermentation profiles of tolerant and sensitive yeast were compared. Some of the strains (YPS606 S.c.; DVBPG6040-S.c.) identified in the study displayed useful phenotypes, such as the tolerance to most of the stress conditions identified in second generation bioethanol fermentations. Hybrids can exhibit more desirable properties than their original parents, an important parameter for future strain development via breeding programmes. Knowledge of the Saccharomyces strains grown in these conditions will aid the development of breeding programmes in order to generate more efficient strains for industrial fermentations. Use of this data will aid increased economic output and the establishment of a viable second-generation bioethanol industry.

\section{Methods}

\section{Yeast strains and growth conditions}

Many of the Saccharomyces spp. strains used in this study have been previously described [22,53,54]. Additional isolates tested included two strains of $S$. arboricolus $[20,55,56]$. The majority of the strains analysed in this study were wild isolates [22] that do not contain any gene deletions or auxotrophic markers. The homothallic parent strain of each isolate had been sporulated and the resultant spores dissected and allowed to self-mate to generate homozygous diploids before they were sequenced [22]. Selected representatives of clean lineage $S$. cerevisiae strains were made genetically tractable by deleting $\mathrm{HO}$ and creating an URA3 auxotrophy by deletion [23] Haploids of these were crossed to form heterozygotes with auxotrophic markers as described previously [23,24]. The hybrid $S$. kudriavzevi $1803 \times$ S. cerevisiae Y55 was generated previously [57] and was also utilised for this study.

For vegetative growth, either yeast extract peptone dextrose (YPD) medium [1\% yeast extract (Oxoid); $2 \%$ (w/v) Bacto-peptone (Oxoid); 2\% (w/v); 2\% (w/v) glucose], or 
YNB (Yeast nitrogen base) medium $[0.67 \%(\mathrm{w} / \mathrm{v}) \mathrm{YNB}$ with amino acids and ammonium sulphate; $6 \%(\mathrm{w} / \mathrm{v})$ glucose] were used. For analysis of growth using alternative carbon sources (pentose sugars), glucose was replaced with $2 \%$ xylose. Cultures were cryopreserved in $20 \%$ glycerol at $-80^{\circ} \mathrm{C}$. Most strains can be obtained from the National Collection of Yeast Cultures (NCYC; see http://www.ncyc.co.uk/ for information). All isolates were stored at $-80^{\circ} \mathrm{C}$ in a 96 -well plate format in $20 \%$ glycerol. More detailed information on each strain and species can be found in the Additional file 1: Figure S1.

\section{Phenotypic microarray analysis}

Phenotypic microarray (PM) technology (Biolog, US) assay is based on the detection of metabolic output using a reporter system [58], the reporter system utilises a redox sensitive tetrazolium dye which upon reduction correlates with an increase in metabolic rate of a cell which is oxidizing a carbon source. For PM analysis each individual well contained growth medium consisting of a final concentrations of $0.67 \%(\mathrm{w} / \mathrm{v})$ yeast nitrogen base (YNB) and $6 \%$ $(\mathrm{w} / \mathrm{v})$ glucose as appropriate for the final assay volume of $120 \mu \mathrm{l}$, supplemented with $2.6 \mu \mathrm{l}$ of yeast nutrient supplement mixture $(\mathrm{NS} \times 48-24 \mathrm{mM}$ adenine- $\mathrm{HCl}$, 4.8 mM L-histidine $\mathrm{HCl}$ monohydrate, $48 \mathrm{mM}$ L-leucine, $24 \mathrm{mM}$ L-lysine- $\mathrm{HCl}, 12 \mathrm{mM}$ L-methionine, $12 \mathrm{mM} \mathrm{L-}$ tryptophan and $14.4 \mathrm{mM}$ uracil) and $0.2 \mu \mathrm{l}$ of dye D (Biolog, Hayward, CA, USA). The final volume was made up to $30 \mu \mathrm{l}$ using sterile distilled water. This was made up fresh as a stock sufficient for each experiment and $30 \mu \mathrm{l}$ dispensed to individual microtitre plate wells containing increasing concentrations of the appropriate inhibitors (acetic acid, formic acid, furfural, hydroxymethyl furfural (HMF), levulinic acid and vanillin). Stock solutions $(1 \mathrm{M})$ of aliphatic weak acids such as acetic acid, formic and levulinic acid were prepared using reverse osmosis (RO) sterilised water; furfural, HMF and vanillin were prepared as $1 \mathrm{M}$ stock solutions in 100\% ethanol. A stock solution of $80 \%$ sorbitol (w/v) was prepared and adjusted to generate $10 \%$ and $15 \%(\mathrm{w} / \mathrm{v})$ concentrations in a final volume of $120 \mu \mathrm{L}$. For ethanol, $10 \%(\mathrm{v} / \mathrm{v})$ and $15 \%(\mathrm{v} / \mathrm{v})$ was used to induce ethanol stress. Temperature was adjusted to either $30^{\circ} \mathrm{C}, 35^{\circ} \mathrm{C}$, or $40^{\circ} \mathrm{C}$ and data was taken at 15 min intervals for 96 hours for $30^{\circ} \mathrm{C}$ and $35^{\circ} \mathrm{C}$, and 24 hours for assays at $40^{\circ} \mathrm{C}$. Assays at $40^{\circ} \mathrm{C}$ were limited in terms of time due to the effect of evaporation if measured for 96 hours. Strains were prepared for inoculation onto $\mathrm{PM}$ assay plates as follows. Glycerol stocks stored at $-80^{\circ} \mathrm{C}$ were streaked onto YPD plates to obtain single colonies and incubated at $30^{\circ} \mathrm{C}$ for approximately $48 \mathrm{hrs}$. Two to three colonies from each strain were then patched on a fresh YPD plate and incubated overnight at $30^{\circ} \mathrm{C}$. Cells were then inoculated into sterile water in $20 \times 100 \mathrm{~mm}$ test tubes and adjusted to a transmittance of $62 \%\left(\sim 5 \times 10^{6}\right.$ cells. $\mathrm{ml}^{-1}$ ) using sterile distilled water using turbidometer. Cell suspensions for the inoculums were then prepared by mixing $125 \mu \mathrm{l}$ of these cells and $2.5 \mathrm{ml}$ of IFY buffer ${ }^{\mathrm{TM}}$ (Biolog, USA) and the final volume adjusted to $3 \mathrm{ml}$ using RO sterile distilled water, $90 \mu \mathrm{l}$ of this mix was inoculated to each well in a Biolog 96-well plate. Anaerobic conditions were generated by placing each plate into a PM gas bag (Biolog, Hayward, CA, USA) and vacuum packed using an Audion VMS43 vacuum chamber (Audion Elektro BV, Netherlands).

The OmniLog reader photographs the plates at $15 \mathrm{~min}$ intervals to measure dye conversion, the pixel intensity in each well is then converted to a value reflecting metabolic output. After completion of the run, the data was compiled and exported from the Biolog software and compiled using Microsoft ${ }^{\circ}$ Excel. In all cases, a minimum of three replicate PM assay runs were conducted and the mean values are presented.

Percentage redox signal intensity was calculated by dividing the redox signal intensity value under stress conditions divided by the redox signal intensity under non-stress conditions after $50 \mathrm{hrs}$ incubation, except for thermal stress at $40^{\circ} \mathrm{C}$, where this was calculated using the redox signal intensity values at 24 hours for control and stressed conditions.

Data were transformed according to [39]. In order to eradicate noise a smoothing parameter was employed by sequentially increasing the number of moving average data points until all negative slopes between points had disappeared. A sliding window average of 25 data points was used to smooth the transformed metabolic output curves in order to facilitate comparisons and analysis.

\section{R statistical computing environment}

Data from the $48 \mathrm{hr}$ time points were analysed using $\mathrm{R}$ version 3.01, platform x86_64-w64-mingw32/x64 [59], data converted into comma delimited files and run on a $\mathrm{R}$ workspace, RGui 64 bit is a free to use software for statistical analysis package http://cran.r-project.org/bin/ windows/base/. This package was used to compare sugar utilisation of Saccharomyces spp. yeast strains.

\section{Spot plate assays}

Spot plate tests were performed according to [60] with modifications. Cells were grown overnight in yeast peptone broth (YPD) at $30^{\circ} \mathrm{C}$ with orbital shaking at $150 \mathrm{rpm}$ [61]. One $\mathrm{mL}$ of culture $\left(\mathrm{OD}_{600}=1.0\right)$ was centrifuged for 2 minutes at $17,000 \mathrm{x} \mathrm{g}$ in a swinging rotor centrifuge operated at $4^{\circ} \mathrm{C}$. The resulting pellet was washed three times using sterile distilled water and re-suspended in $100 \mu \mathrm{L}$ of sterile distilled water. Next, the re-suspended cells were diluted to an initial $\mathrm{OD}_{600}$ of 1 , serially diluted, and a $5 \mu \mathrm{L}$ aliquot from each 10-fold dilution 
was spotted onto agar plates containing $0.67 \% \mathrm{YNB}+6 \%$ glucose and incubated at $35^{\circ} \mathrm{C}$ and $40^{\circ} \mathrm{C}$ for 3 days, no inhibitory compounds were added.

\section{Confirmation of phenotypic microarray results using mini fermentation vessels}

Fermentations were conducted in $180 \mathrm{~mL}$ mini-fermentation vessels (FV). Cryopreserved yeast colonies were streaked onto YPD plates and incubated at $30^{\circ} \mathrm{C}$ for $48 \mathrm{hrs}$. Colonies of yeast strains YPS606, and DBVPG1583 were used to inoculate $20 \mathrm{ml}$ of YPD broth and incubated in an orbital shaker at $30^{\circ} \mathrm{C}$ for 24 hrs. These were then transferred to $200 \mathrm{~mL}$ of YPD and grown for $48 \mathrm{hrs}$ in a $500 \mathrm{ml}$ conical flask shaking at $30^{\circ} \mathrm{C}$. Cells were harvested and washed three times with sterile RO water and then resuspended in $5 \mathrm{ml}$ of sterile water. For control conditions, $1.5 \times 10^{7}$ cells. $\mathrm{mL}^{-1}$ were inoculated in $99.6 \mathrm{ml}$ of medium containing $4 \%$ glucose, $2 \%$ peptone, $1 \%$ yeast extract with $0.4 \mathrm{ml}$ RO water. For stress conditions, $1.5 \times$ $10^{7}$ cells. $\mathrm{mL}^{-1}$ were incubated in $99.6 \mathrm{ml}$ of medium containing $4 \%$ glucose, $2 \%$ peptone, $1 \%$ yeast extract with $10 \mathrm{mM}$ acetic acid, $5 \mathrm{mM}$ formic acid, $5 \mathrm{mM}$ levulinic acid, $5 \mathrm{mM}$ furfural, $5 \mathrm{mM} \mathrm{HMF}$ and $5 \mathrm{mM}$ vanillin. Volumes of media were adjusted to account for the addition of the inhibitory compounds $(\sim 400 \mu \mathrm{L})$ to ensure that all fermentations began with the same carbon load.

Anaerobic conditions were prepared using a sealed butyl plug (Fisher, Loughborough, UK) and aluminium caps (Fisher Scientific). A hypodermic needle attached with a Bunsen valve was purged through rubber septum to facilitate the release of $\mathrm{CO}_{2}$. All experiments were performed in triplicate and weight loss was measured at each time point. Mini-fermentations were conducted at $35^{\circ} \mathrm{C}$, with orbital shaking at $200 \mathrm{rpm}$.

\section{Hierarchical clustering analysis of fermentation stress conditions}

The hierarchical clustering algorithm used is based closely on the average-linkage method of Sokal and Michener, 1958 , the object of this algorithm is to compute a dendrogram that assembles all elements into a single tree $[2,62]$. The matrix is scanned to identify the highest value (representing the most similar pair of strains) in comparison with the reference yeast strain $S$. cerevisiae S288C. Gene Cluster 3.0 was used to construct matrices [63] which are compatible with TreeView for production of representative dendrograms $[63,64]$.

\section{Additional files}

Additional file 1: Figure S1. Effect of temperature on growth of S. uvarum. Cells were grown on YNB with $6 \%(\mathrm{w} / \mathrm{v})$, under aerobic conditions at a variety of incubation temperatures for 36 hours. S. cerevisiae (S288C) was added as a reference strain. Figure S2. Comparison of sensitive (DBVPG1853) and tolerant (YPS606) S. cerevisiae strains to 15\% sorbitol,

$10 \%$ ethanol, $35^{\circ} \mathrm{C}$ and $40^{\circ} \mathrm{C}, 25 \mathrm{mM}$ acetic acid, $10 \mathrm{mM}$ formic acid, $10 \mathrm{mM}$ levulinic acid, $10 \mathrm{mM} \mathrm{HMF}, 10 \mathrm{mM}$ furfural and $10 \mathrm{mM}$ vanillin individually using phenotypic microarray analysis. Data expressed as\% RSI of the unstressed wells. Figure S3. Number of Saccharomyces strains identified as more tolerant than the parent strains utilised to produce hybrids under sorbitol, formic acid and temperature stress, metabolic output for S. cerevisiae (Y12), S. cerevisiae (YPS128), S. cerevisiae (Y55) and S. kudriavzevii (IFO1803) for $10 \%$ (w/v) sorbitol, $5 \mathrm{mM}$ formic acid and $35^{\circ} \mathrm{C}$.

Additional file 2: Phenotypic microarray analysis (redox signal intensity) for Saccharomyces spp. at 25 hour time point, data in the file is tabulated in the following order 1 - Strain details and background information, 2 - Percentage redox signal of the control values for all stress conditions, 3 - Ranking of the strains for osmotic stress, 4 - Ranking of the strains for ethanol stress, 5 - Ranking of the strains for thermal stress- $35^{\prime} \mathrm{C}$, 6 - Ranking of the strains for thermal stress- $40^{\prime} C, 7$ - Ranking of the strains for fermentation stress, 8 - Ranking of the strains for acetic acid, 9 - Ranking of the strains for formic acid, 10 - Ranking of the strains for levulinic acid, 11 - Ranking of the strains for HMF, 12 - Ranking of the strains for furfural, 13 - Ranking of the strains for vanillin, 14 - Ranking of the strains for vanillin and HMF, 15 - Ranking of the strains for the inhibitors, 16 - Ranking of the strains for all fermentation stress. The data shown is an average of triplicate values.

\section{Abbreviations}

LCM: Lignocellulosic material; PM: Phenotypic microarray; NS: Nutrient supplement.

\section{Competing interests}

The authors declare that they have no competing interest.

\section{Authors' contributions}

TW and DG equally contributed to work including generating data and writing the manuscript. MM and GL helped in designing experiments. GL donated some of the hybrid strains and EL donated the sensu stricto yeast. $\mathrm{YC}$ and $\mathrm{AH}$ helped in collecting data and MM helped in processing it. MM, GT, TP, EL and KS have proof read the manuscript. All authors read and approved the final manuscript.

\section{Acknowledgements}

The research reported here was supported (in full or in part) by the Biotechnology and Biological Sciences Research Council (BBSRC) Sustainable Bioenergy Centre (BSBEC), under the programme for 'Lignocellulosic Conversion to Ethanol' (LACE) [Grant Ref: BB/G01616X/1]. This is a large interdisciplinary programme and the views expressed in this paper are those of the authors alone, and do not necessarily reflect the views of the collaborators or the policies of the funding bodies. This project is part financed by the European Regional Development Fund project EMX05568.

\section{Author details}

${ }^{1}$ Bioenergy \& Brewing Science, School of Biosciences, Sutton Bonington Campus, University of Nottingham, Loughborough, Leicestershire LE12 6RD, UK. ${ }^{2}$ Centre for Genetic Architecture of Complex Traits, Department of Genetics, University of Leicester, Adrian Building, Leicester LE1 7RH, UK. ${ }^{3}$ Institute for Research on Cancer and Aging, Faculty of Medicine, 28, Avenue De Valombrose, 06107 Nice, Cedex-02, France. ${ }^{4}$ Current address: SABMiller plc, SABMiller House. Church Street West, Woking, Surrey GU21 6HS, UK. ${ }^{5}$ Pepsico Int, 4, Leycroft Road, Leicester LE4 1ET, UK.

Received: 12 December 2013 Accepted: 19 March 2014 Published: 27 March 2014

\section{References}

1. Logan BE, Rabaey K: Conversion of wastes into bioelectricity and chemicals by using microbial electrochemical technologies. Science 2012, 337:686-690.

2. Soccol CR, Vandenberghe LPD, Medeiros ABP, Karp SG, Buckeridge M, Ramos $L P$, Pitarelo AP, Ferreira-Leitao V, Gottschalk LMF, Ferrara MA, da Silva Bon EP, de Moraes LM, Araujo JDA, Torres FA: Bioethanol from lignocelluloses: status and perspectives in Brazil. Bioresour Technol 2010, 101:4820-4825. 
3. Amorim HV, Lopes ML, Oliveira JVD, Buckeridge MS, Goldman GH: Scientific challenges of bioethanol production in Brazil. Appl Microbiol Biotechnol 2011, 91:1267-1275.

4. Campbell JE, Block E: Land-use and alternative bioenergy pathways for waste biomass. Environ Sci Technol 2010, 44:8665-8669.

5. Leal MRLV, Nogueira LAH, Cortez LAB: Land demand for ethanol production. Appl Energy 2013, 102:266-271.

6. Sims RE, Mabee W, Saddler JN, Taylor M: An overview of second generation biofuel technologies. Bioresour Technol 2010, 101:1570-1580.

7. Laluce C, Schenberg AC, Gallardo JC, Coradello LF, Pombeiro-Sponchiado SR: Advances and developments in strategies to improve strains of Saccharomyces cerevisiae and processes to obtain the lignocellulosic ethanol-a review. Appl Biochem Biotechnol 2012, 166:1908-1926.

8. Harcus D, Dignard D, Lepine G, Askew C, Raymond M, Whiteway M, Wu C: Comparative xylose metabolism among the ascomycetes C. albicans, S. stipitis and S. cerevisiae. PLOS One 2013, 8:e80733.

9. Wang $C$, Shen $Y$, Zhang $Y$, Suo F, Hou J, Bao X: Improvement of $\mathrm{L}$-arabinose fermentation by modifying the metabolic pathway and transport in Saccharomyces cerevisiae. Biomed Res Int 2013, 2013:461204.

10. Kotter PCM: Xylose fermentation by Saccharomyces cerevisiae. Appl Microbiol Biotechnol 1993, 38:776-783.

11. Attfield PV, Bell PJ: Use of population genetics to derive nonrecombinant Saccharomyces cerevisiae strains that grow using xylose as a sole carbon source. FEMS Yeast Res 2006, 6:862-868.

12. Wenger JW, Schwartz K, Sherlock G: Bulk segregant analysis by highthroughput sequencing reveals a novel xylose utilization gene from Saccharomyces cerevisiae. PLoS Genet 2010, 6:e1000942.

13. Kuyper M, Winkler AA, van Dijken JP, Pronk JT: Minimal metabolic engineering of Saccharomyces cerevisiae for efficient anaerobic xylose fermentation: a proof of principle. FEMS Yeast Res 2004, 4:655-664.

14. Zhao XQ, Bai FW: Mechanisms of yeast stress tolerance and its manipulation for efficient fuel ethanol production. J Biotechnol 2009, 144:23-30.

15. Jonsson $\sqcup$, Alriksson B, Nilvebrant NO: Bioconversion of lignocellulose: inhibitors and detoxification. Biotechnol Biofuels 2013, 6:16.

16. Palmqvist E, Grage $\mathrm{H}$, Meinander NQ, Hahn-Hagerdal B: Main and interaction effects of acetic acid, furfural, and p-hydroxybenzoic acid on growth and ethanol productivity of yeasts. Biotechnol Bioeng 1999, 63:46-55.

17. Libkind D, Hittinger CT, Valerio E, Goncalves C, Dover J, Johnston M, Goncalves P, Sampaio JP: Microbe domestication and the identification of the wild genetic stock of lager-brewing yeast. Proc Natl Acad Sci U S A 2011, 108:14539-14544.

18. Liti G, Ba ANN, Blythe M, Muller CA, Bergstrom A, Cubillos FA, Dafhnis-Calas F, Khoshraftar S, Malla S, Mehta N, Siow CC, Warringer J, Moses AM, Louis EJ, Nieduszynski CA: High quality de novo sequencing and assembly of the Saccharomyces arboricolus genome. BMC Genomics 2013, 14. doi:10.1186/ 1471-2164-14-69.

19. Louis EJ: Population genomics and speciation in yeasts. Fungal Biol Rev 2011, 25:136-142.

20. Naumov Gl, Naumova ES, Masneuf-Pomarede I: Genetic identification of new biological species Saccharomyces arboricolus Wang et Bai. Antonie Van Leeuwenhoek 2010, 98:1-7.

21. Naumova ES, Naumov GI, Masneuf-Pomarede I, Aigle M, Dubourdieu D: Molecular genetic study of introgression between Saccharomyces bayanus and S. cerevisiae. Yeast 2005, 22:1099-1115.

22. Liti G, Carter DM, Moses AM, Warringer J, Parts L, James SA, Davey RP, Roberts IN, Burt A, Koufopanou V, Tsae IJ, Bergman CM, Bensasson D, O'Kelly MJ, van Oudenaarden A, Barton DB, Bailes E, Nguyen AN, Jones M, Quail MA, Goodhead I, Sims S, Smith F, Blomberg A, Durbin R, Louis EJ: Population genomics of domestic and wild yeasts. Nature 2009, 458:337-341.

23. Cubillos FA, Louis El, Liti G: Generation of a large set of genetically tractable haploid and diploid Saccharomyces strains. FEMS Yeast Res 2009, 9:1217-1225.

24. Cubillos FA, Billi E, Zorgo E, Parts L, Fargier P, Omholt S, Blomberg A, Warringer Louis EJ, Liti G: Assessing the complex architecture of polygenic traits in diverged yeast populations. Mol Ecol 2011, 20:1401-1413.

25. Parts L, Cubillos FA, Warringer J, Jain K, Salinas F, Bumpstead SJ, Molin M, Zia A, Simpson JT, Quail MA, Moses A, Louis EJ, Durbin R, Liti G: Revealing the genetic structure of a trait by sequencing a population under selection. Genome Res 2011, 21:1131-1138.

26. Belloch COS, Barrio E, Querol A: Fermentative stress adaptation of hybrids within the Saccharomyces sensu stricto complex. Int J Food Microbiol 2008, 29:188-195.
27. Fleet GH: Wine yeasts for the future. FEMS Yeast Res 2008, 8:979-995.

28. Masneuf I, Hansen J, Groth C, Piskur J, Dubourdieu D: New hybrids between Saccharomyces sensu stricto yeast species found among wine and cider production strains. Appl Environ Microbiol 1998, 64:3887-3892.

29. Replansky T, Koufopanou V, Greig D, Bell G: Saccharomyces sensu stricto as a model system for evolution and ecology. Trends Ecol Evol 2008, 23:494-501.

30. Cakar ZP, Turanli-Yildiz B, Alkim C, Yilmaz U: Evolutionary engineering of Saccharomyces cerevisiae for improved industrially important properties. FEMS Yeast Res 2012, 12:171-182.

31. Benjaphokee S, Hasegawa D, Yokota D, Asvarak T, Auesukaree C, Sugiyama M, Kaneko Y, Boonchird C, Harashima S: Highly efficient bioethanol production by a Saccharomyces cerevisiae strain with multiple stress tolerance to high temperature, acid and ethanol. N Biotechnol 2012, 29:379-386.

32. Saerens SG, Duong CT, Nevoigt E: Genetic improvement of brewer's yeast: current state, perspectives and limits. Appl Microbiol Biotechnol 2010, 86:1195-1212.

33. Pereira FB, Guimaraes PMR, Gomes DG, Mira NP, Teixeira MC, Sa-Correia I, Domingues L: Identification of candidate genes for yeast engineering to improve bioethanol production in very high gravity and lignocellulosic biomass industrial fermentations. Biotechnol Biofuels 2011, 4. doi:10.1186/ 1754-6834-4-57.

34. Tomas-Pejo E, Oliva JM, Ballesteros M, Olsson L: Comparison of SHF and SSF processes from steam-exploded wheat straw for ethanol production by xylose-fermenting and robust glucose-fermenting Saccharomyces cerevisiae strains. Biotechnol Bioeng 2008, 100:1122-1131.

35. Kunicka-Styczynska A, Rajkowska K: Phenotypic and genotypic diversity of wine yeasts used for acidic musts. World J Microbiol Biotechnol 2012, 28:1929-1940.

36. Favaro L, Basaglia M, Trento A, van Rensburg E, Garcia-Aparicio M, van Zyl $\mathrm{WH}$, Casella S: Exploring grape marc as trove for new thermotolerant and inhibitor-tolerant Saccharomyces cerevisiae strains for second-generation bioethanol production. Biotechnol Biofuels 2013, 6:168.

37. Kumari R, Pramanik K: Improvement of multiple stress tolerance in yeast strain by sequential mutagenesis for enhanced bioethanol production. J Biosci Bioeng 2012, 114:622-629.

38. Larsson S, PAlmqvist E, Hahn-Hagerdal B, Tengborg C, Stenberg K, Zacchi G, Nilvebrant $\mathrm{N}$ : The generation of fermentation inhibitors during dilute acid hydrolysis of softwood. Enzyme Microb Technol 1999, 24:151-159.

39. Warringer J, Zorgo E, Cubillos FA, Zia A, Gjuvsland A, Simpson JT, Forsmark A, Durbin R, Omholt SW, Louis EJ, Liti G, Moses A, Blomberg A: Trait variation in yeast is defined by population history. PLoS Genet 2011, 7:e1002111.

40. Powell CD, Quain DE, Smart KA: The impact of brewing yeast cell age on fermentation performance, attenuation and flocculation. FEMS Yeast Res 2003, 3:149-157.

41. Fay JC, Benavides JA: Evidence for domesticated and wild populations of Saccharomyces cerevisiae. PLoS Genet 2005, 1:66-71.

42. Briggs DEB, Boulton CA, Brookes PA, Stevens R: Brewing Science and practice. Cambridge: Woodhead Publishing Limited; 2004

43. Casey GP, Ingledew WM: Ethanol tolerance in yeasts. Crit Rev Microbiol 1986, 13:219-280.

44. Sonderegger M, Jeppsson M, Larsson C, Gorwa-Grauslund MF, Boles E, Olsson L, Spencer-Martins I, Hahn-Hagerdal B, Sauer U: Fermentation performance of engineered and evolved xylose-fermenting Saccharomyces cerevisiae strains. Biotechnol Bioeng 2004, 87:90-98.

45. Zheng DQ WP, Chen J, Zhang K, Liu TZ, Wu XC, Li YD, Zhao YH: Genome sequencing and genetic breeding of a bioethanol Saccharomyces cerevisiae strain YJS329. BMC Genomics 2012, 13. doi:10.1186/1471-2164-13-479.

46. Goncalves P, Valerio E, Correia C, de Almeida JM, Sampaio JP: Evidence for divergent evolution of growth temperature preference in sympatric Saccharomyces species. PLoS One 2011, 6:e20739.

47. Rainieri S, Zambonelli C, Kaneko Y: Saccharomyces sensu stricto: systematics, genetic diversity and evolution. J Biosci Bioeng 2003, 96:1-9.

48. Salvadó ZA-LF, Guillamón JM, Salazar G, Querol A, Barrio E: Temperature adaptation markedly determines evolution within the genus Saccharomyces. Appl Environ Microbiol 2011, 77:2292-2302.

49. Marinoni G, Manuel M, Petersen RF, Hvidtfeldt J, Sulo P, Piskur J: Horizontal transfer of genetic material among Saccharomyces yeasts. J Bacteriol 1999, 181:6488-6496.

50. Katou T, Namise M, Kitagaki H, Akao T, Shimoi H: QTL mapping of sake brewing characteristics of yeast. J Biosci Bioeng 2009, 107:383-393. 
51. Lin $Y$, Tanaka S: Ethanol fermentation from biomass resources: current state and prospects. Appl Microbiol Biotechnol 2006, 69:627-642.

52. Zorgo E, Gjuvsland A, Cubillos FA, Louis EJ, Liti G, Blomberg A, Omholt SW, Warringer J: Life history shapes trait heredity by accumulation of loss-of-function alleles in yeast. Mol Biol Evol 2012, 29:1781-1789.

53. Liti G, Barton DB, Louis EJ: Sequence diversity, reproductive isolation and species concepts in Saccharomyces. Genetics 2006, 174:839-850.

54. Liti G, Peruffo A, James SA, Roberts IN, Louis EJ: Inferences of evolutionary relationships from a population survey of LTR-retrotransposons and telomeric-associated sequences in the Saccharomyces sensu stricto complex. Yeast 2005, 22:177-192.

55. Naumov Gl: Hybridization analysis of the new biological species Saccharomyces arboricolus Wang et Bai. Dokl Biol Sci 2009, 426:247-249.

56. Wang S-A, Bai F-Y: Saccharomyces arboricolus sp. nov., a yeast species from tree bark. Int I Syst Evol Microbiol 2008, 58:510-514.

57. Greig D, Borts RH, Louis EJ, Travisano M: Epistasis and hybrid sterility in Saccharomyces. Proc Biol Sci Royal Soc 2002, 269:1167-1171.

58. Bochner BR: Sleuthing out bacterial identities. Nature 1989, 339:157-158.

59. Team RDC: R: A language and environment forstatistical computing. Vienna, Austria: R Foundation for Statistical Computing; 2008.

60. Homann OR, Cai H, Becker JM, Lindquist SL: Harnessing natural diversity to probe metabolic pathways. PLoS Genet 2005, 1:e80.

61. Hohmann S: Osmotic stress signaling and osmoadaptation in Yeasts. Microbiol Mol Biol Rev 2002, 66:300-372.

62. Sokal RR, Michener CD: A statistical method for evaluating systematic relationships. Univ Kans Sci Bull 1958, 38:1409-1442.

63. Eisen MB, Spellman PT, Brown PO, Botstein D: Cluster analysis and display of genome-wide expression patterns. Proc Natl Acad Sci U S A 1998, 95:14863-14868.

64. Saldanha AJ: Java Treeview-extensible visualization of microarray data. Bioinformatics 2004, 20:3246-3248.

doi:10.1186/1475-2859-13-47

Cite this article as: Wimalasena et al:: Phenotypic characterisation of Saccharomyces spp. yeast for tolerance to stresses encountered during fermentation of lignocellulosic residues to produce bioethanol. Microbial Cell Factories 2014 13:47.

\section{Submit your next manuscript to BioMed Central and take full advantage of:}

- Convenient online submission

- Thorough peer review

- No space constraints or color figure charges

- Immediate publication on acceptance

- Inclusion in PubMed, CAS, Scopus and Google Scholar

- Research which is freely available for redistribution 